\title{
Telavancin in the Treatment of Concurrent Staphylococcus aureus Bacteremia: A Retrospective Analysis of ATLAS and ATTAIN Studies
}

Samuel E. Wilson · Donald R. Graham - Whedy Wang •

Jon B. Bruss · Bibiana Castaneda-Ruiz

Received: March 14, 2017 / Published online: July 10, 2017

(C) The Author(s) 2017. This article is an open access publication

\begin{abstract}
Introduction: Concurrent Staphylococcus aureus bacteremia (SAB) worsens outcomes and increases mortality in patients with complicated skin and skin structure infections (cSSSI), hospital-acquired bacterial pneumonia, and ventilator-associated bacterial pneumonia (HABP/ VABP). These challenges highlight the need for alternative treatments. Telavancin (TLV), a bactericidal lipoglycopeptide with high in vitro potency, effectively treats patients with cSSSI and HABP/VABP caused by Gram-positive pathogens, particularly $S$. aureus.

Methods: This retrospective analysis evaluated patients from the Assessment of Telavancin in Complicated Skin and Skin Structure Infections and Assessment of Telavancin for Treatment of
\end{abstract}

Enhanced content To view enhanced content for this article go to http://www.medengine.com/Redeem/ C0A8F060507851E1.

S. E. Wilson

University of California, Irvine, CA, USA

D. R. Graham

Springfield Clinic, Springfield, IL, USA

W. Wang · J. B. Bruss · B. Castaneda-Ruiz $(\bowtie)$

Theravance Biopharma US, Inc., South San

Francisco, CA, USA

e-mail: BCastaneda@theravance.com
Hospital-Acquired Pneumonia studies with baseline, concurrent SAB. Differences in the clinical cure rates at test-of-cure and safety outcomes were compared for TLV vs vancomycin (VAN) treatment groups.

Results: A total of 105 patients, 32 cSSSI and 73 HABP/VABP, had baseline, concurrent SAB. The clinical cure rates for all-treated $S A B$ patients in the cSSSI (TLV 57.1\% and VAN 54.5\%) and HABP/VABP (TLV 54.3\% and VAN 47.2\%) groups were comparable. For both types of infections, the safety profile of TLV and VAN showed similar incidences of adverse events (AEs), serious AEs, or AEs leading to discontinuation. One VAN-treated patient died in the cSSSI group, and there were 13 deaths in each treatment arm of the HABP/VABP group.

Conclusion: This retrospective analysis demonstrated that TLV is clinically comparable in both efficacy and safety to VAN, and, therefore, may be an appropriate therapeutic option for the treatment of patients with $\mathrm{HABP} / \mathrm{VABP}$ or cSSSI and concurrent SAB. Given the limited sample size in this subgroup, the interpretation of these results is limited.

Funding: Theravance Biopharma Antibiotics, Inc.

Keywords: Complicated skin and skin structure infection; Concurrent bacteremia; MRSA; Pneumonia; Staphylococcus aureus; Telavancin; Vancomycin 


\section{INTRODUCTION}

Staphylococcus aureus causes a wide range of infections, including hospital-acquired bacterial pneumonia (HABP), ventilator-associated bacterial pneumonia (VABP), complicated skin and skin structure infections (cSSSI), and bacteremia [1]. Primary bacteremia that occurs without a prior bacterial entry portal or associated site of infection accounts for approximately $40 \%-50 \%$ of cases of $S$. aureus bacteremia (SAB) [2]. SAB that occurs with an established portal of entry or associated infection, such as HABP/VABP or cSSSI, is defined as secondary or concurrent bacteremia [2]. Several studies have reported $S$. aureus to be a common pathogen in patients with HABP/VABP or cSSSI and concurrent bacteremia [3-8]. The global incidence rates of concurrent bacteremia in HABP/VABP or cSSSI patients range from $12 \%-20 \%[3-7,9,10]$. Concurrent $\mathrm{SAB}$ is associated with complications including infective endocarditis, septic arthritis, and osteomyelitis, and a delay in treatment of the primary infection further increases the risk of developing these complications [2]. Furthermore, patients with HABP/ VABP or cSSSI and concurrent bacteremia have poor outcomes and, in some cases, increased mortality compared with patients without bacteremia [3-8]. Given the severity of illness and higher mortality rates observed in patients with concurrent $\mathrm{SAB}$, there is a need to identify alternative treatments.

Telavancin (TLV) is a bactericidal lipoglycopeptide antibiotic with a dual mechanism of action; it inhibits cell wall synthesis and disrupts the functional integrity of the bacterial membrane [11] and has high in vitro potency. It is active against certain clinically relevant Gram-positive pathogens, including methicillin-susceptible and methicillin-resistant $S$. aureus (MSSA, MRSA) [12]. The Assessment of Telavancin in Complicated Skin and Skin Structure Infections (ATLAS) and Assessment of Telavancin for Treatment of Hospital-Acquired Pneumonia (ATTAIN) trials have demonstrated that TLV is noninferior to vancomycin (VAN) in the treatment of patients with cSSSIs and HABP/ VABP caused by Gram-positive pathogens
$[12,13]$. Telavancin is currently approved in the US in adults for the treatment of cSSSI due to susceptible Gram-positive pathogens, and for HABP/VABP caused by susceptible isolates of $S$. aureus when other treatments are not suitable. The retrospective efficacy and safety analysis of patients from the ATLAS and ATTAIN trials presented here demonstrates that TLV may be an appropriate therapeutic option in treating patients with either cSSSI or HABP/VABP who have concurrent SAB. In May 2016, the US Food and Drug Administration approved the inclusion of portions of these data in the TLV US product label.

\section{METHODS}

\section{Subject Selection}

Patients enrolled in the ATLAS and ATTAIN studies (ClinicalTrials.gov Identifiers: NCT00091819, NCT00107978, NCT00107952, NCT00124020) who had concurrent bacteremia were selected for this retrospective analysis $[12,13]$. Concurrent bacteremia in cSSSI and $\mathrm{HABP} / \mathrm{VABP}$ patients was defined as the recovery of $S$. aureus from the baseline blood culture. This article is based on previously conducted studies and does not involve any new studies of human or animal subjects performed by any of the authors.

\section{Antimicrobial Treatment Regimen}

The ATLAS and ATTAIN trials were double-blinded studies wherein patients were randomized to receive either intravenous TLV $(10 \mathrm{mg} / \mathrm{kg}$ every $24 \mathrm{~h})$ or VAN ( $1 \mathrm{~g}$ every $12 \mathrm{~h}$ ) for 7-14 days (CSSSI) or 7-21 days (HABP/VABP) $[12,13]$. For both infection types, TLV dose was adjusted as per renal function and VAN dose was administered per institutional protocol [14]. For the ATTAIN and ATLAS trials, initial VAN dosage ranged from $125-4000 \mathrm{mg}$ and 143-3400 mg every $24 \mathrm{~h}$, respectively. There was no sequential oral treatment allowed. The modified all-treated population included all randomized patients who received $\geq 1$ dose of 
study drug with pathogen identified from baseline samples $[12,13]$.

\section{Efficacy and Safety Variables and Statistical Analyses}

The clinical response of each patient was determined by the investigator at the end of treatment and at the follow-up assessment. For the cSSSI patients, clinical cure was defined as resolution of clinically significant signs and symptoms present at study admission or improvement to the extent that the infectious process had been controlled and no further antimicrobial therapy was needed [13]. For HABP/VABP patients, clinical cure was defined as improvement or lack of progression of baseline radiographic findings at end of therapy and resolution of signs and symptoms of pneumonia at follow-up/test-of-cure [12]. For both infection types, for purposes of analysis, a clinical response of "Not Cured" at end of treatment was carried forward to test-of-cure (TOC). Clinical cure rates at TOC were calculated as the number of patients with concurrent $\mathrm{SAB}$ and a clinical response of "cure" divided by the total number of patients with concurrent SAB. Creatinine clearance $(\mathrm{CrCl})$ was estimated using the Cockcroft-Gault equation [15]. Baseline renal function was stratified into 4 categories according to $\mathrm{CrCl}$, $<30,30-50,>50-80$, and $>80 \mathrm{~mL} / \mathrm{min}$.

For both types of infection, 28-day mortality was defined as any death that occurred within 28 days after end of treatment [12, 13]. Post hoc analyses across treatment groups consisted of an estimated treatment difference (stratified by study) with its accompanying 95\% confidence interval (CI) adjusted via Agresti-Caffo methods, as appropriate [16]. For each approved indication, the incidence of treatment-emergent adverse events (AEs) and serious AEs (SAEs) were descriptively recorded. Adverse events are reported in terms defined by the Medical Dictionary for Regulatory Activities (MedDRA) unless otherwise specified.

\section{Compliance with Ethics Guidelines}

This article is based on previously conducted studies and does not involve any new studies of human or animal subjects performed by any of the authors.

\section{RESULTS}

\section{Patient Disposition, Demographic, and Baseline Characteristics}

A total of 105 patients, 32 cSSSI (21 TLV and 11 VAN) and 73 HABP/VABP (35 TLV and 38 VAN) patients from the ATLAS and ATTAIN studies, respectively, had concurrent SAB. Baseline and demographic variables were comparable for both treatment groups within each infection type (Table 1 ). Patients aged $\geq 65$ years accounted for approximately $31 \%$ of the cSSSI and 55\% of the HABP/VABP groups. For both cSSSI and HABP/VABP groups with concurrent bacteremia, common comorbidities included diabetes and hypertension. The baseline pathogens isolated for each infection are also listed in Table 1. Impaired renal function $(\mathrm{CrCl}$ levels $\leq 50 \mathrm{~mL} / \mathrm{min}$ ) was observed at baseline in $20 \%$ and $31 \%$ of patients with cSSSI and HABP/ VABP, respectively. Approximately equal numbers of patients with HABP/VABP and concurrent SAB were ventilated at baseline in the TLV $(n=18,51 \%)$ compared with VAN $(n=19$, $50 \%)$ groups. In cSSSI patients with concurrent bacteremia, the $S$. aureus (MRSA and MSSA) minimum inhibitory concentration for $50 \%$ or $90 \%$ of isolates $\left(\mathrm{MIC}_{50}, \mathrm{MIC}_{90}\right.$ ) were $0.06 \mu \mathrm{g} / \mathrm{mL}$ for $\mathrm{TLV}$ and $1 \mu \mathrm{g} / \mathrm{mL}$ for VAN, respectively. Among HABP/VABP patients with bacteremia, the MSSA MIC $_{50}$ and MIC $_{90}$ values were 0.03 and $0.06 \mu \mathrm{g} / \mathrm{mL}$ for TLV, respectively, and $1 \mu \mathrm{g} /$ mL for VAN; the MRSA MIC 50 and MIC $_{90}$ values were 0.06 and $0.12 \mu \mathrm{g} / \mathrm{mL}$ for TLV, respectively, and $1 \mu \mathrm{g} / \mathrm{mL}$ for VAN.

\section{Efficacy Outcomes}

Telavancin-treated patients with cSSSI and concurrent SAB received their study drug for a mean of 10 days (median of 8 days, range of 1-15 days), and VAN-treated patients received their study drug for a mean of 9 days (median of 10 days, range of $2-15$ days). Overall, 21 (66\%) 
Table 1 Baseline and demographic characteristics

\begin{tabular}{|c|c|c|c|c|}
\hline \multirow[t]{2}{*}{ Characteristic } & \multicolumn{2}{|c|}{ ATLAS-cSSSI [13] } & \multicolumn{2}{|c|}{ ATTAIN-HABP/VABP [12] } \\
\hline & $\begin{array}{l}\text { TLV } \\
n=21\end{array}$ & $\begin{array}{l}\text { VAN } \\
n=11\end{array}$ & $\begin{array}{l}\text { TLV } \\
n=35\end{array}$ & $\begin{array}{l}\text { VAN } \\
n=38\end{array}$ \\
\hline \multicolumn{5}{|l|}{ Age } \\
\hline Mean years \pm SD & $52 \pm 16.9$ & $52 \pm 19.4$ & $65 \pm 20.1$ & $61 \pm 17.6$ \\
\hline$<65$ years, $n(\%)$ & $15(71)$ & $7(64)$ & $13(37)$ & $20(53)$ \\
\hline$\geq 65$ years, $n(\%)$ & $6(29)$ & $4(36)$ & $22(63)$ & $18(47)$ \\
\hline \multicolumn{5}{|l|}{ Race, $n(\%)$} \\
\hline White & $16(76)$ & $8(73)$ & $25(71)$ & $26(68)$ \\
\hline African American & $3(14)$ & $3(27)$ & $2(6)$ & $2(5)$ \\
\hline Asian & $2(10)$ & $0(0)$ & $8(23)$ & $7(18)$ \\
\hline American Indian or Alaskan native & $0(0)$ & $0(0)$ & $0(0)$ & $3(8)$ \\
\hline Weight, mean $\mathrm{kg} \pm \mathrm{SD}$ & $75 \pm 15.7$ & $73 \pm 15.6$ & $73 \pm 16.3$ & $72 \pm 18.6$ \\
\hline Body mass index, mean $\mathrm{kg} / \mathrm{m}^{2} \pm \mathrm{SD}$ & $26 \pm 4.4$ & $26 \pm 5.0$ & $26 \pm 5.4$ & $25 \pm 5.7$ \\
\hline \multicolumn{5}{|l|}{ S. aureus, $n(\%)$} \\
\hline Infection site, MSSA & $6(29)$ & $4(36)$ & $12(34)$ & $10(26)$ \\
\hline Infection site, MRSA & $11(52)$ & $5(4)$ & $17(49)$ & $24(63)$ \\
\hline Blood, MSSA & $8(38)$ & $7(64)$ & $14(40)$ & $14(37)$ \\
\hline Blood, MRSA & $13(62)$ & $4(36)$ & $21(60)$ & $24(63)$ \\
\hline \multicolumn{5}{|c|}{ Baseline renal function $(\mathrm{CrCl} \mathrm{mL} / \mathrm{min}), n(\%)$} \\
\hline$>80$ & $11(55)$ & $4(40)$ & $15(43)$ & $14(42)$ \\
\hline$>50-80$ & $6(30)$ & $3(30)$ & $11(31)$ & $7(21)$ \\
\hline $30-50$ & $3(15)$ & $2(20)$ & $6(17)$ & $7(21)$ \\
\hline$<30$ & $0(0)$ & $1(10)$ & $3(9)$ & $5(15)$ \\
\hline Diabetes, $n(\%)$ & $6(29)$ & $3(27)$ & $6(17)$ & $10(26)$ \\
\hline Hypertension, $n$ (\%) & $9(43)$ & $5(45)$ & $20(57)$ & $18(47)$ \\
\hline
\end{tabular}

ATLAS Assessment of Telavancin in Complicated Skin and Skin Structure Infections, ATTAIN Assessment of Telavancin for Treatment of Hospital-Acquired Pneumonia, $\mathrm{CrCl}$ creatinine clearance, $c S S S I$ complicated skin and skin structure infections, $H A B P / V A B P$ hospital-acquired and ventilator-associated bacterial pneumonia, $M R S A$ methicillin-resistant Staphylococcus aureus, MSSA methicillin-susceptible Staphylococcus aureus, SD standard deviation, TLV telavancin, VAN vancomycin

cSSSI bacteremic patients (13 TLV and 8 VAN) completed the course of study drug therapy and had resolution of signs and symptoms in $\leq 14$ days. A total of 11 ( $8 \mathrm{TLV}$ and 3 VAN) patients discontinued treatment, with unsatisfactory therapeutic response being the most common reason for drug discontinuation. Overall clinical cure rates were similar between TLV-treated patients $(12 / 21,57.1 \%)$ and VAN-treated patients $(6 / 11,54.5 \%)$ [difference: 
Table 2 Clinical cure rates at test-of-cure in patients with bacteremia in ATLAS and ATTAIN trials

\begin{tabular}{|c|c|c|c|c|c|c|}
\hline \multirow{2}{*}{$\begin{array}{l}\text { Pathogen } \\
\text { identified } \\
\text { in the blood }\end{array}$} & \multicolumn{3}{|c|}{ ATLAS-cSSSI [13] } & \multicolumn{3}{|c|}{ ATTAIN-HABP/VABP [12] } \\
\hline & $\begin{array}{l}\overline{T L V} \\
n=21\end{array}$ & $\begin{array}{l}\text { VAN } \\
n=11\end{array}$ & $\begin{array}{l}\text { Differer } \\
\text { (95\% C }\end{array}$ & $\begin{array}{l}\text { TLV } \\
n=35\end{array}$ & $\begin{array}{l}\text { VAN } \\
n=38\end{array}$ & $\begin{array}{l}\text { Difference }^{a} \\
(95 \% \mathrm{CI})\end{array}$ \\
\hline . & $12 / 21(57.1)$ & $6 / 11(54.5)$ & $-0.8(-34.4,35.5)^{\mathrm{b}}$ & $19 / 35(54.3)$ & $18 / 38(47.4)$ & $9.9(-13.9,33.6)$ \\
\hline MSSA & $4 / 8$ & $4 / 7(57.1)$ & $-6.0(-48.4,40.4)^{\mathrm{b}}$ & $8 / 14$ & $9 / 14$ & $-6.5(-39.7,29.8)^{b}$ \\
\hline MRSA & $8 / 13(61.5)$ & $2 / 4(50.0)$ & $-0.5(-44.3,52.2)^{\mathrm{b}}$ & $11 / 21(52.4)$ & $9 / 24(37.5)$ & $17.7(-12.8,42.3)^{\mathrm{b}}$ \\
\hline
\end{tabular}

Unless otherwise noted, all data are presented as the number of cured patients/total number of patients in the specific group, and the percentage is in parentheses

ATLAS Assessment of Telavancin in Complicated Skin and Skin Structure Infections, ATTAIN Assessment of Telavancin for Treatment of Hospital-Acquired Pneumonia, CI confidence interval, $c S S S I$ complicated skin and skin structure infections, $H A B P / V A B P$ hospital-acquired and ventilator-associated bacterial pneumonia, MRSA methicillin-resistant Staphylococcus aureus, MSSA methicillin-susceptible Staphylococcus aureus, TLV telavancin, VAN vancomycin

${ }^{a}$ Pooled analysis stratified by study. Cure rate is calculated as the number of patients with the given pathogen and a clinical response of "cure" divided by the number of patients with the given pathogen

b $95 \%$ CI for the treatment difference (TLV-VAN) in cure rate using Agresti-Caffo adjustment for sparse data

$-0.8 \% \quad(95 \% \quad$ CI $-34.4 \%, 35.5 \%)]$ (Table 2). Eight patients ( $7 \mathrm{TLV}, 1 \mathrm{VAN})$ had a clinical response "not cured," 2 patients (1 TLV, 1 VAN) had an indeterminate response, and 4 ( $1 \mathrm{TLV}, 3$ VAN) had a missing response at TOC. Treatment failure was most commonly attributed to drug discontinuation due to unsatisfactory therapeutic response. One VAN-treated patient with concurrent bacteremia died in the cSSSI studies. The patient presented with cellulitis at the peripheral intravenous infusion site at baseline, developed hypoxemia due to pulmonary edema, and ultimately died of septic shock.

Three cSSSI patients had concurrent bacteremia (2 MSSA and 1 MRSA) that persisted for 3,4 , and 6 days past the baseline blood culture, respectively. Two were treated with VAN, and 1 was treated with TLV. The TLV-treated patient was also diagnosed with infective endocarditis and vertebral osteomyelitis. All 3 patients with persistent bacteremia were considered treatment failures.

Telavancin-treated patients with $\mathrm{HABP} /$ VABP and concurrent $\mathrm{SAB}$ received the study drug for a mean of 11 days (median of 11 , range of 2-22 days), and VAN-treated patients received the study drug for a mean of 9 days (median of 9 , range of 1-23 days). Overall, 38 (52\%) HABP/VABP patients with SAB (19 TLV and 19 VAN) completed the course of study drug therapy and had resolution of signs and symptoms in $\leq 21$ days. A total of 35 (16 TLV and $19 \mathrm{VAN}$ ) patients discontinued treatment with unsatisfactory therapeutic response being the most common reason for drug discontinuation. The overall clinical cure rates for the TLV $(54.3 \%)$ and VAN (47.4\%) groups were comparable (difference of $9.9 \%$ [95\% CI $-13.9 \%$, 33.6\%]) (Table 2). Twelve patients (4 TLV, 8 VAN) had a clinical response "failure," 5 patients ( $2 \mathrm{TLV}, 3 \mathrm{VAN}$ ) had an indeterminate response, and 19 (10 TLV, 9 VAN) had a missing response at TOC. Treatment failure was most commonly attributed to progression of pneumonia. In total, 29 deaths were reported in the $\mathrm{HABP} / \mathrm{VABP}$ bacteremic patients; the 28-day, all-cause mortality rate was comparable in the 2 treatment groups (TLV 14/35 [40.0\%] and VAN $15 / 38$ [39.5\%]).

In the HABP/VABP studies, 4 patients had bacteremia that persisted beyond baseline in the TLV group (1 MSSA and 3 MRSA). Two of the 4 patients were cured after 3 and 4 days of positive blood cultures, respectively. The other 2 patients died; 1 due to septic shock after 7 days of persistent positive blood cultures for MRSA, and the other patient had care withdrawn after 5 days of persistent positive blood cultures. Eight VAN-treated patients had persistent bacteremia (2 MSSA and 6 MRSA). One of these patients, with 3 days of blood cultures 
positive for MRSA, was cured. Five of the other 7 patients died, all following 3-4 days of persistent positive blood cultures. The remaining 2 patients, who were bacteremic for 6 and 7 days, respectively, were successfully treated with linezolid or a combination of cloxacillin and clindamycin, respectively.

\section{Safety Analysis}

For the cSSSI patients, no notable difference was observed between the treatment groups in the incidences of AEs, SAEs, or AEs leading to discontinuation of study drug. A total of $2(6 \%)$ cSSSI patients with concurrent SAB (TLV 1/21 and VAN 1/11) discontinued study medication due to AEs. Common AEs, such as dysgeusia, headache, vomiting, and foamy urine, were reported more often in the TLV group (Table 3). There was a single report each of renal impairment and renal insufficiency. These MedDRA-defined AEs were reported by the investigators based on their clinical judgment. No renal AEs were reported in the VAN treatment group.

The frequencies and types of AEs, SAEs, and AEs leading to discontinuation of the study drug were comparable between treatment groups for HABP/VABP patients with concurrent SAB. A total of 7 (10\%) bacteremic patients (TLV 2/35 and VAN 5/38) discontinued the study drug due to AEs. Anemia, septic shock, and hypokalemia were the most commonly reported AEs by the HABP/VABP bacteremic patients in both treatment groups (Table 3). The incidence of renal AEs was less than 10\% across both treatments in HABP/VABP patients with concurrent SAB (TLV, 2 renal impairment and 1 renal insufficiency; VAN, 2 acute renal failure, 1 renal insufficiency, and 1 nephrotic syndrome).

\section{DISCUSSION}

HABP/VABP or cSSSI with concurrent bacteremia is a significant issue often caused by $S$. aureus [3-10]. However, data for the treatment of bacteremia, concurrent with HABP/VABP or cSSSI, are limited and warrant further study. While ceftaroline demonstrated efficacy in treating 48 patients with $\mathrm{SAB}$ concurrent with acute bacterial skin and skin structure infections or community-acquired bacterial pneumonia [17], its efficacy in treating concurrent $S A B$ in HABP/VABP patients has not been evaluated. Alternative therapies are needed for SAB, especially for patients with bacteremia secondary to infections that tend to have worse clinical outcomes, including HABP/VABP or cSSSI [3-8].

In considering evidence for the potential efficacy of TLV in SAB, outcomes for patients with concurrent bacteremia in the phase 3 ATLAS (CSSSI) and ATTAIN (HABP/VABP) studies were retrospectively examined [12, 13]. Thirty-two of 1867 (1.7\%) and 73 of 1503 (4.9\%) patients had concurrent bacteremia from the overall ATLAS and ATTAIN populations, respectively (Table 1$)[12,13]$. The difference in bacteremia incidence between cSSSI and HABP/ VABP infections could relate to the varying vascularity of the infected tissues and organs. For example, subcutaneous fat has less blood flow than lung parenchyma. As noted in other studies, $S$. aureus was the most common Gram-positive pathogen isolated from blood and primary infection site samples (Table 1) [3-10]. For both infection types, the clinical cure rates at TOC for patients with concurrent $\mathrm{SAB}$ were comparable for VAN and TLV treatments (Table 2).

As bacteremic patients tend to be sicker and are associated with poorer outcomes, safety outcomes were examined in this patient subset. The safety data for this population were similar to the overall ATLAS and ATTAIN population. Among patients with concurrent SAB, the safety profile of TLV was similar to that of VAN, with comparable incidences of AEs, SAEs, or AEs leading to discontinuation, and were comparable between the treatment groups. Renal AEs were uncommon $(\leq 10 \%)$ for both treatment groups in the cSSSI ( 2 TLV and 0 VAN) and HABP/VABP (3 TLV and 4 VAN) studies. Cases of persistent bacteremia were noted in both the cSSSI (1 TLV and 1 VAN) and HABP/VABP (4 TLV and 12 VAN) patients. However, as the ATLAS and ATTAIN studies were not designed to capture persistent bacteremia as an endpoint, these cases cannot be further evaluated. One VAN-treated patient died in the cSSSI group, 
Table 3 Common adverse events $(\geq 10 \%)$

Adverse event
(system organ class preferred term), $n$ (\%)

\section{ATLAS-cSSSI [13]}

\begin{tabular}{ll}
\hline TLV & VAN \\
$n=21$ & $n=11$
\end{tabular}

$2(10)$

$2(10)$

$2(10)$

$3(14)$

4 (19)

7 (33)

4 (19)

Vomiting
ATTAIN-HABP/VABP [12]

TLV VAN

$n=35$

$n=38$
$2(5)$
$0(0)$
$1(3)$

$1(3)$

$0(0)$

$4(11)$

$3(8)$

3 (9)

$3(8)$

1 (9)

$6(17)$

1 (3)

4 (36)

$5(14)$

$0(0)$

General disorders and administration site conditions

Edema peripheral

$1(9)$

$4(11)$

Infections and infestations

Pneumonia

$1(5)$

$0(0)$

$1(3)$

$4(11)$

Septic shock

$0(0)$

$1(9)$

$4(11)$

$5(13)$

Metabolism and nutrition disorders

Hypokalemia

$0(0)$

$5(14)$

$4(11)$

Hypomagnesemia

$1(5)$

2 (18)

$1(3)$

$2(5)$

Nervous system disorders

Dysgeusia

6 (29)

1 (9)

$3(27)$

$0(0)$

$1(3)$

Psychiatric disorders

Agitation

Anxiety

Insomnia

Renal and urinary disorders

Foamy urine $^{\mathrm{a}}$

$4(19)$

$2(10)$

$0(0)$

Dyspnea

$0(0)$

$0(0)$

Respiratory, thoracic, and mediastinal disorders

Pulmonary edema
$0(0)$

$0(0)$

$1(9)$

$4(19)$

$\begin{array}{ll}2(10) & 0(0) \\ 2(10) & 0(0) \\ 4(19) & 1(9)\end{array}$

$2(6)$

$2(5)$

$1(3)$

$1(3)$ 
Table 3 continued

\begin{tabular}{|c|c|c|c|c|}
\hline \multirow{2}{*}{$\begin{array}{l}\text { Adverse event } \\
\text { (system organ class preferred term), } n \text { (\%) }\end{array}$} & \multicolumn{2}{|c|}{ ATLAS-cSSSI [13] } & \multicolumn{2}{|c|}{ ATTAIN-HABP/VABP [12] } \\
\hline & $\begin{array}{l}\text { TLV } \\
n=21\end{array}$ & $\begin{array}{l}\text { VAN } \\
n=11\end{array}$ & $\begin{array}{l}\operatorname{TLV} \\
n=35\end{array}$ & $\begin{array}{l}\text { VAN } \\
n=38\end{array}$ \\
\hline \multicolumn{5}{|l|}{ Skin and subcutaneous tissue disorders } \\
\hline Pruritus & $1(5)$ & $2(18)$ & $0(0)$ & $1(3)$ \\
\hline Pruritus generalized & $1(5)$ & $2(18)$ & - & - \\
\hline \multicolumn{5}{|l|}{ Vascular disorders } \\
\hline Hypotension & $0(0)$ & $2(18)$ & $2(6)$ & $4(11)$ \\
\hline
\end{tabular}

$A E$ adverse event, ATLAS Assessment of Telavancin in Complicated Skin and Skin Structure Infections, ATTAIN Assessment of Telavancin for Treatment of Hospital-Acquired Pneumonia, cSSSI complicated skin and skin structure infections, $H A B P / V A B P$ hospital-acquired and ventilator-associated bacterial pneumonia, MedDRA Medical Dictionary for Regulatory Activities, $T L V$ telavancin, $V A N$ vancomycin

- The AE was not reported in either trial of the respective study

a Three patients reported 'foamy urine' and 1 patient reported 'frothy urine'; the MedDRA term for these reports is 'urine abnormality'

whereas the 26 deaths in the HABP/VABP group were equally distributed among both treatments. The high mortality rate (approximately $40 \%$ ) experienced by HABP/VABP patients with concurrent bacteremia [12] is consistent with other pneumonia studies that observed greater mortality in bacteremic HABP/VABP patients than in patients without bacteremia $[3,8]$.

This post hoc analysis has several limitations. First, it is retrospective. Second, it has a small subset of patients with concurrent bacteremia [12, 13] compared with other reports $[3,5,7,10]$. Third, in the ATLAS and ATTAIN studies, the patients were not stratified by the presence of bacteremia; therefore, prognostic factors, such as persistence of infection, time to defervescence, and metastatic foci, were not evaluated. Fourth, this analysis was limited to patients who had positive blood cultures and infection site samples at baseline, and, therefore, excluded patients who may have developed bacteremia during the course of these trials. Finally, only 7-14 or 7-21 days of therapy was given to patients in the ATLAS and ATTAIN studies, respectively, which precludes a comparison of the effectiveness of a longer duration of therapy. Duration of therapy is an area of $\mathrm{SAB}$ management that lacks robust evidence [18].

\section{CONCLUSION}

Telavancin is a bactericidal antimicrobial agent with high in vitro potency that effectively treats cSSSI and HABP/VABP infections caused by Gram-positive pathogens [12, 13]. This retrospective analysis of the cSSSI and HABP/VABP bacteremic patients from the ATLAS and ATTAIN studies, respectively, demonstrates that TLV is comparable to VAN and may be a viable alternative to VAN for treatment of cSSSI or $\mathrm{HABP} / \mathrm{VABP}$ with concurrent SAB.

\section{ACKNOWLEDGEMENTS}

This study and the article processing charges were supported by Theravance Biopharma Antibiotics, Inc., George Town, Cayman Islands. All authors had full access to all of the data in this study and take complete responsibility for the integrity of the data and accuracy of the data analysis. The authors acknowledge Drs. Anna Osmukhina and Steven L. Barriere for their input and critical review of this manuscript. Editorial support in the preparation and submission of this manuscript was provided by Tanmayi Mankame, PhD; and Nicole Seneca, 
PhD, of AlphaBioCom, LLC, King of Prussia, PA, USA, and was funded by Theravance Biopharma Antibiotics, Inc. All named authors meet the International Committee of Medical Journal Editors (ICMJE) criteria for authorship for this manuscript, take responsibility for the integrity of the work as a whole, and have given final approval for the version to be published.

Disclosures. Dr. Castaneda is an employee of Theravance Biopharma US, Inc. Dr. Wang is an employee of Theravance Biopharma US, Inc. Dr. Bruss is a former employee of Theravance Biopharma US, Inc. His current affiliation is Alarus Development International. Dr. Wilson reports advisory board and lecture fees from BrioMed outside the submitted work. Dr. Graham reports grants from Theravance during the conduct of the study.

Compliance with Ethics Guidelines. This article is based on previously conducted studies and does not involve any new studies of human or animal subjects performed by any of the authors.

Data Availability. The datasets generated during and/or analyzed during the current study are available from the corresponding author upon reasonable request.

Open Access. This article is distributed under the terms of the Creative Commons Attribution-NonCommercial 4.0 International License (http://creativecommons.org/licenses/ by-nc/4.0/), which permits any noncommercial use, distribution, and reproduction in any medium, provided you give appropriate credit to the original author(s) and the source, provide a link to the Creative Commons license, and indicate if changes were made.

\section{REFERENCES}

1. Tong SY, Davis JS, Eichenberger E, Holland TL, Fowler VG Jr. Staphylococcus aureus infections: epidemiology, pathophysiology, clinical manifestations, and management. Clin Microbiol Rev. 2015;28(3):603-61.
2. del Rio A, Cervera C, Moreno A, Moreillon P, Miro JM. Patients at risk of complications of Staphylococcus aureus bloodstream infection. Clin Infect Dis. 2009;48(Suppl 4):S246-53.

3. Agbaht K, Diaz E, Munoz E, Lisboa T, Gomez F, Depuydt PO, et al. Bacteremia in patients with ventilator-associated pneumonia is associated with increased mortality: a study comparing bacteremic vs. nonbacteremic ventilator-associated pneumonia. Crit Care Med. 2007;35(9):2064-70.

4. Kunac A, Sifri ZC, Mohr AM, Horng H, Lavery RF, Livingston DH. Bacteremia and ventilator-associated pneumonia: a marker for contemporaneous extra-pulmonic infection. Surg Infect (Larchmt). 2014;15(2):77-83.

5. O'Keefe GE, Caldwell E, Cuschieri J, Wurfel MM, Evans HL. Ventilator-associated pneumonia: bacteremia and death after traumatic injury. J Trauma Acute Care Surg. 2012;72(3):713-9.

6. Ranes JL, Gordon SM, Chen P, Fatica C, Hammel J, Gonzales JP, et al. Predictors of long-term mortality in patients with ventilator-associated pneumonia. Am J Med. 2006;119(10):897 e13-9.

7. Jaaskelainen IH, Hagberg L, From J, Schyman T, Lehtola L, Jarvinen A. Treatment of complicated skin and skin structure infections in areas with low incidence of antibiotic resistance- a retrospective population based study from Finland and Sweden. Clin Microbiol Infect. 2016;22(4):383.e1-10.

8. Magret M, Lisboa T, Martin-Loeches I, Manez R, Nauwynck M, Wrigge $H$, et al. Bacteremia is an independent risk factor for mortality in nosocomial pneumonia: a prospective and observational multicenter study. Crit Care. 2011;15(1):1-8.

9. Gursel G, Demir N. Incidence and risk factors for the development of acute renal failure in patients with ventilator-associated pneumonia. Nephrology (Carlton). 2006;11(3):159-64.

10. McClure JR, Cooke RPD, Lal P, Pickles D, Majjid S, Grant CA, et al. Outcome of late-onset hospital-acquired pneumonia related to causative organism. J Hosp Infect. 2009;71(4):348-52.

11. Higgins DL, Chang R, Debabov DV, Leung J, Wu T, Krause KM, et al. Telavancin, a multifunctional lipoglycopeptide, disrupts both cell wall synthesis and cell membrane integrity in methicillin-resistant Staphylococcus aureus. Antimicrob Agents Chemother. 2005;49(3):1127-34.

12. Rubinstein E, Lalani T, Corey GR, Kanafani ZA, Nannini EC, Rocha MG, et al. Telavancin versus vancomycin for hospital-acquired pneumonia due 
to gram-positive pathogens. Clin Infect Dis. 2011;52(1):31-40.

13. Stryjewski ME, Graham DR, Wilson SE, O'Riordan $\mathrm{W}$, Young D, Lentnek A, et al. Telavancin versus vancomycin for the treatment of complicated skin and skin-structure infections caused by gram-positive organisms. Clin Infect Dis. 2008;46(11):1683-93.

14. VIBATIV $^{\circledR}$ (telavancin), USP [package insert]. South San Francisco, CA: Theravance Biopharma US. 2016.

15. Cockcroft DW, Gault MH. Prediction of creatinine clearance from serum creatinine. Nephron. 1976;16(1):31-41.
16. Agresti A, Caffo B. Simple and effective confidence intervals for proportions and differences of proportions result from adding two successes and two failures. Am Stat. 2000;54(4):280-8.

17. Vazquez JA, Maggiore CR, Cole P, Smith A, Jandourek A, Friedland HD. Ceftaroline fosamil for the treatment of bacteremia secondary to acute bacterial skin and skin structure infections or community-acquired bacterial pneumonia. Infect Dis Clin Pract (Baltim Md). 2015;23(1):39-43.

18. Holland TL, Arnold C, Fowler VG Jr. Clinical management of Staphylococcus aureus bacteremia: a review. JAMA. 2014;312(13):1330-41. 